\section{Evaluación comparativa entre MELD y Child-Pugh como escalas pronósticas de sobrevida en pacientes con cirrosis hepática en Chile}

\author{
EDGAR SANHUEZA ${ }^{1,4}$, JORGE CONTRERAS ${ }^{1,2,4}$, RODRIGO ZAPATA ${ }^{1,3}$, \\ MATÍAS SANHUEZA ${ }^{3, a}$, FABIÁN ELGUETA ${ }^{2}$, CONSTANZA LÓPEZ $^{3}$, \\ SIGRID JEREZ ${ }^{1, b}$, VERÓNICA JEREZ ${ }^{1, b}$, IRIS DELGADO ${ }^{4, c}$
}

\section{Comparative evaluation of survival prognosis using MELD or Child-Pugh scores in patients with liver cirrhosis in Chile}

Background: Currently, most liver units use the Child-Pugh (CP) or the Model for End-Stage Liver Disease (MELD) scores to establish survival prognosis among patients with liver cirrhosis. Which classification is superior, is not well defined. Aim: To compare CP and MELD classification scores to predict survival among adult patients with liver cirrhosis in Chile. Material and Methods: Follow-up of 137 consecutive adult patients with liver cirrhosis aged $59 \pm 12$ years (55\% women). The diagnosis was reached by clinical, laboratory and image studies at three different centers of Santiago. Patients were staged with CP and MELD classification scores at baseline and followed over a period of 12 months. The predictive capacity of the scores for survival was analyzed using a multivariate statistical analysis (Kaplan-Meier curves). Results: The most common etiology was alcohol (37.9\%). The actuarial survival rate was $79.6 \%$ at 12 months of follow-up. When comparing groups with areas under curve of receiver operating characteristic curves (AUROC), there was no statistically significant difference in survival between less severe and advanced disease, assessed with both survival scales. The AUROC for MELD and CP were 0.80 and 0.81, respectively. Conclusions: This clinical study did not find a statistically significant difference between the two classifications for the prediction of 12 months survival in patients with cirrhosis.

(Rev Med Chile 2017; 145: 17-24)

Key words: Liver Cirrhosis; Liver Transplantation; Survival Rate; ROC Curve.
'Unidad de Gastroenterología. Departamento de Medicina Interna. Clínica Alemana de Santiago. Santiago, Chile. ${ }^{2}$ Departamento de Medicina Interna. Hospital Padre Hurtado. Santiago, Chile.

${ }^{3}$ Departamento de Medicina

Oriente. Sección de

Gastroenterología. Hospital del

Salvador. Facultad de Medicina,

Sede Oriente, Universidad de

Chile. Santiago, Chile.

${ }^{4}$ Facultad de Medicina

Universidad del Desarrollo.

Santiago, Chile.

aAlumno de Medicina, Campus

Oriente, Universidad de Chile.

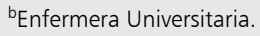

'Bioestadística.

Fuente de apoyo: No.

Recibido el 3 de abril de 2016, aceptado el 19 de noviembre de 2016.

Correspondencia a:

Edgar Sanhueza Bravo

Avenida Vitacura 5951, Santiago,

Chile.

Teléfono: + 56222106032

esanhueza@alemana.cl
L a cirrosis hepática es una enfermedad crónica de alta prevalencia en el mundo, su etiología es variable, dependiente de la localización geográfica ${ }^{1}$, su pronóstico está determinado por el grado de insuficiencia hepática y la presencia de complicaciones al momento del diagnóstico. El único tratamiento curativo que existe en la actualidad para pacientes con enfermedad hepática avan- zada y terminal es el trasplante de hígado $(\mathrm{TH})$.

El desarrollo de la técnica quirúrgica, un mejor manejo en unidades de cuidados intensivos y el uso de nuevos fármacos inmunosupresores han aumentado la sobrevida del paciente sometido a TH y del injerto, con una considerable disminución de las complicaciones ${ }^{2,3}$. El TH se plantea como una opción adecuada cuando la sobrevida 
que conlleva el procedimiento es mayor que la esperada utilizando tratamiento médico ${ }^{4,5}$. Por esta razón, conocer el pronóstico de sobrevida de esta enfermedad es fundamental, y para esto es necesario utilizar escalas pronósticas validadas, las cuales permiten determinar a quienes y cuando trasplantar ${ }^{6,7}$.

Tradicionalmente se ha utilizado la clasificación de Child-Pugh (CP) para establecer pronóstico en cirrosis $^{8-10}$, el rango de esta escala varía entre 5 y 15 puntos, basado en la presencia de ascitis, encefalopatía y parámetros de laboratorio (bilirrubina, albúmina y protrombina), estableciéndose las clases A, B y C. Esta escala fue inicialmente utilizada en pacientes cirróticos que iban a cirugía de shunt porto-sistémico, siendo posteriormente validada en otros escenarios clínicos ${ }^{11}$.

En el año 2000, Malinchoc y cols. establecen la clasificación de MELD (Model for End-Stage Liver Disease), para predecir sobrevida en pacientes con cirrosis hepática sometidos a la instalación de un TIPS (Transjugular Intrahepatic Portosystemic Shunt $)^{12}$. A partir del año 2002, esta escala comienza a ser usada en Estados Unidos de Norteamérica (EE.UU.) para la asignación y priorización de órganos en $\mathrm{TH}^{13-16}$. Este modelo predictivo (MELD) se ha extendido y ha sido utilizado para el pronóstico de sobrevida en cirrosis de cualquier etiología ${ }^{17,18,19}$. Actualmente es de elección en varios centros y países para establecer prioridad en la asignación de órganos en $\mathrm{TH}^{20,21}$. Desde el año 2012, y por consenso de todos los grupos que realizan TH en Chile, se estableció MELD como el método nacional para asignación de órganos.

Existen numerosas publicaciones en la literatura que han intentado establecer la superioridad de MELD sobre la escala de CP, sin embargo, hasta la fecha no existe claridad sobre cuál podría ser el mejor método para determinar el pronóstico de la enfermedad $^{22-29}$. La clasificación de CP considera cinco variables estableciendo 3 categorías (A, B y $C$ ) en la cual A es la más leve y $C$ la más grave. Por otra parte, la clasificación de MELD considera sólo tres variables (creatinina, bilirrubina e INR), determina sobrevida a 3 meses y utiliza una escala numérica continua. Nuestro objetivo es comparar ambas escalas y determinar si existe superioridad en la capacidad pronóstica de sobrevida de una sobre la otra en pacientes chilenos. En Chile no se han realizado estudios clínicos que analicen este tema.

\section{Material y Método}

Entre el 1 de mayo de 2010 y el 30 de diciembre de 2012, se realizó seguimiento a 144 pacientes adultos cirróticos atendidos en forma ambulatoria u hospitalizada en los Servicios de Medicina Interna del Hospital Padre Hurtado, Hospital del Salvador y Clínica Alemana de Santiago. Estos pacientes se enrolaron de manera consecutiva. El diagnóstico de cirrosis hepática se basó en la historia clínica, estudios de laboratorio, imágenes o histología, cuando estaba disponible según criterios habituales. Todos los pacientes aceptaron firmar un consentimiento informado aprobado por el Comité de Ética de los tres centros pertinentes. Se consideraron dentro de los criterios de exclusión a pacientes cirróticos con patologías que disminuyeran significativamente su sobrevida a corto plazo, se excluyen pacientes con: hepatocarcinoma, síndrome hepatorenal, hemorragia digestiva alta variceal o peritonitis bacteriana espontánea en las 2 semanas previas al reclutamiento; pacientes con comorbilidad asociada relevante (cáncer, insuficiencia cardiaca o respiratoria avanzada); patologías o terapias que afectaran la coagulación (ej. uso de anticoagulantes); y patología crónica o psiquiátrica que impidiese manifestar su voluntad de participar en el estudio. Una vez reclutados, se efectuó una encuesta simple que incluyó lo siguiente: nombre completo, R.U.T, edad, género, dirección y teléfono. Se consignó en una base de datos: la etiología de la cirrosis hepática, presencia de ascitis y encefalopatía y parámetros de laboratorio (bilirrubina, creatinina e INR). Una vez terminado el seguimiento, todas las variables de identificación del paciente fueron anonimizadas, de tal manera que el análisis estadístico se realizó teniendo presente la no trasgresión de la Ley 19.628 de privacidad de los datos. Se aplicó la clasificación de CP modificada ${ }^{8}$, que incluye parámetros de laboratorio y clínicos. La clasificación de MELD fue calculada de acuerdo a la fórmula propuesta por Kamath: $3,8 * \log$ (bilirrubina $[\mathrm{mg} / \mathrm{dl}]+$ $11,2 * \ln (I N R)+9,6 * \ln ($ creatinina $[m g / d l]+6,4\}$ $(\text { los valores menores a } 1 \text { se aproximaron a } 1)^{12}$.

Se efectuó seguimiento a 12 meses de ingresados al estudio a través de ficha clínica, encuesta telefónica o información de registro civil. Los datos fueron registrados y analizados en programa estadístico SPSS versión 22.0 (Illinois, USA). El análisis estadístico incluyó una descripción de 
variables a través de frecuencias absolutas y relativas para las variables categóricas y estadísticas de tendencia central para las variables continuas. Para la comparación de las curvas Child y MELD se implementó un análisis de sobrevida Kaplan y Miers verificando la significancia estadística a través del estadígrafo Log-Rank $(\mathrm{p}<0,05)$. A través de curvas ROC se estimó el valor predictivo de cada escala.

Del total de 144 pacientes evaluados para este estudio: uno no aceptó participar; cuatro fueron excluidos por datos relevantes incompletos en el registro y dos no completaron el seguimiento. De esta forma, la cohorte final fue de 137 pacientes.

Este trabajo cumple con las normas de ética establecidas por la Declaración de Helsinki (2008) para estudio de investigación.

\section{Resultados}

De los 137 pacientes que completaron el seguimiento, 75 fueron mujeres $(54,7 \%)$ y 62 hombres $(45,3 \%)$. La edad promedio fue de 59,2 $\pm 12,2$ años (rango 18-80 años).

Veinticuatro pacientes fueron atendidos en Clínica Alemana de Santiago (17,5\%), 58 se enrolaron en Hospital Padre Hurtado (42,3\%) y 60 pacientes en el Hospital del Salvador $(43,8 \%)$.

Las etiologías más frecuentes fueron: alcohol $(\mathrm{n}=52 / 137 ; 37,9 \%)$, hígado graso no alcohólico $(\mathrm{n}=21 / 137 ; 15,3 \%)$, hepatitis $\mathrm{C}(\mathrm{n}=20 / 137$; $14,6 \%)$, hepatitis autoinmune ( $\mathrm{n}=19 / 137$; $13,9 \%)$, cirrosis biliar primaria $(n=9 / 137 ; 6,6 \%)$, criptogénica $(\mathrm{n}=8 / 137 ; 5,8 \%)$, otras etiologías y no definido ( $\mathrm{n}=8 / 137 ; 5,8 \%)$ (Tabla 1$)$.

Para el análisis y comparación entre escalas de sobrevida, los pacientes fueron subdivididos en grupos según los siguientes criterios: La clasificación de $\mathrm{CP}$ se dividió en grupos A ( $<6$ puntos), $\mathrm{B}$ ( 7 a 9 puntos) y C ( $>$ de 9 puntos). La clasificación de MELD se dividió en grupo I ( $<$ o igual a 15 puntos), Grupo II (16-20 puntos) y Grupo III (> de 20 puntos).

La curva de sobrevida global actuarial para la cohorte total fue de 79,6\% al año de seguimiento (Figura 1).La sobrevida del grupo CP fue la siguiente: grupo A $(96,8 \%), \mathrm{B}(72,1 \%), \mathrm{C}(54,8 \%)$ diferencia estadísticamente significativa (LogRank global $<0,0001$ ) (Figura 2). La sobrevida del grupo MELD fue la siguiente: grupo I (86,7\%), II
Tabla 1. Características demográficas del grupo en estudio

\begin{tabular}{|c|c|c|}
\hline Variable & $\mathbf{n}$ & $\%$ \\
\hline \multicolumn{3}{|l|}{ Sexo } \\
\hline Hombres & 62 & 45,3 \\
\hline Mujeres & 75 & 54,7 \\
\hline \multicolumn{3}{|l|}{ Edad (años) } \\
\hline $18-49$ & 24 & 17,5 \\
\hline $50-64$ & 62 & 45,3 \\
\hline $65-80$ & 51 & 37,2 \\
\hline \multicolumn{3}{|l|}{ Etiología } \\
\hline Alcohol & 52 & 37,9 \\
\hline Virus $C$ & 20 & 14,6 \\
\hline Esteatohepatitis no $\mathrm{OH}$ & 21 & 15,3 \\
\hline Criptogénica & 8 & 5,8 \\
\hline Autoinmune & 19 & 13,9 \\
\hline Cirrosis biliar & 9 & 6,6 \\
\hline Otras y no definido & 8 & 5,8 \\
\hline \multicolumn{3}{|l|}{ Muerte } \\
\hline No & 109 & 79,6 \\
\hline \multirow[t]{2}{*}{ Sí } & 28 & 20,4 \\
\hline & Promedio (DE) & Rango \\
\hline Edad (años) & $59,2( \pm 12,2)$ & $18-80$ \\
\hline Meld & $12,6 \quad( \pm 5,2)$ & $6-30$ \\
\hline Child & $7,4 \quad( \pm 2,3)$ & $5-13$ \\
\hline
\end{tabular}

(70\%), III (33,3\%). (Log-Rank entre grupo I y II fue de 0,05 y grupo I y III $<0,001$ respectivamente) (Figura 3).

Al comparar ambas clasificaciones mediante el análisis de curvas de sobrevida se obtuvo un área bajo la curva de $0,81 \%$ para $\mathrm{CP}$ y $0,80 \%$ para MELD respectivamente (Figuras 4 y 5 ).

\section{Discusión}

Cada año fallecen en Chile un poco más de 4.000 personas por complicaciones de la cirrosis. Según un estudio nacional colaborativo con 3.153 pacientes cirróticos evaluados, la etiología alcohólica fue la causa más frecuente, seguida de virus $C$. En nuestro país existen muchos pacientes esperando un $\mathrm{TH}$, con una tasa de donación baja $y$ alta mortalidad en lista de espera ${ }^{30,31}$. Para su enlistamiento y trasplante se utiliza la clasificación de MELD, la cual considera sólo variables objetivas, lo que la hace más práctica y menos variable que la de CP, debido a que esta última utiliza variables subjetivas (grado de encefalopatía y severidad de la 


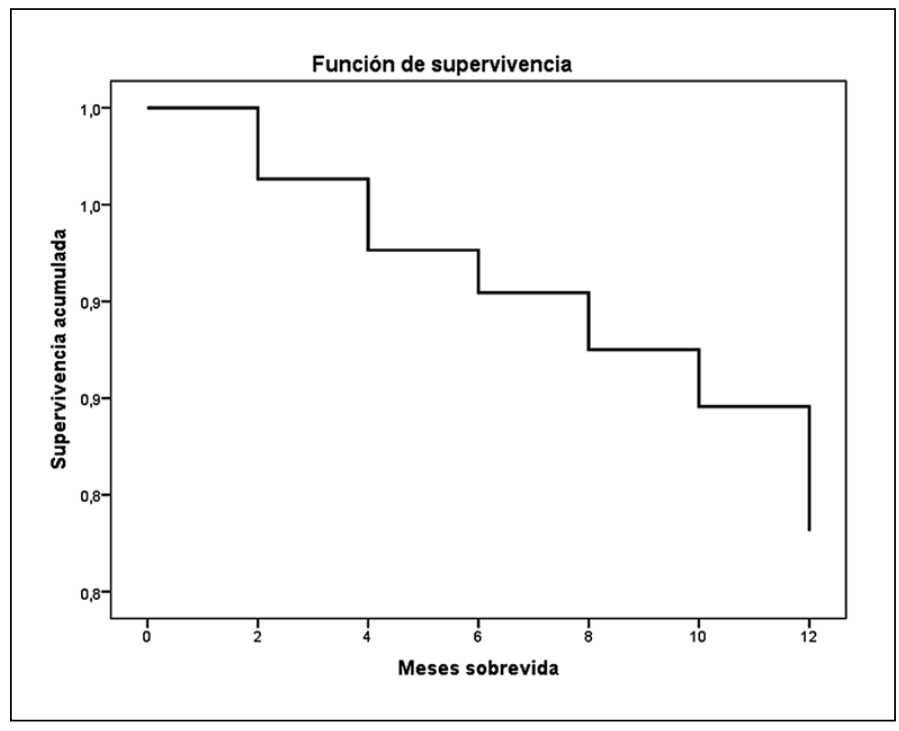

Figura 1. Sobrevida global de la muestra.

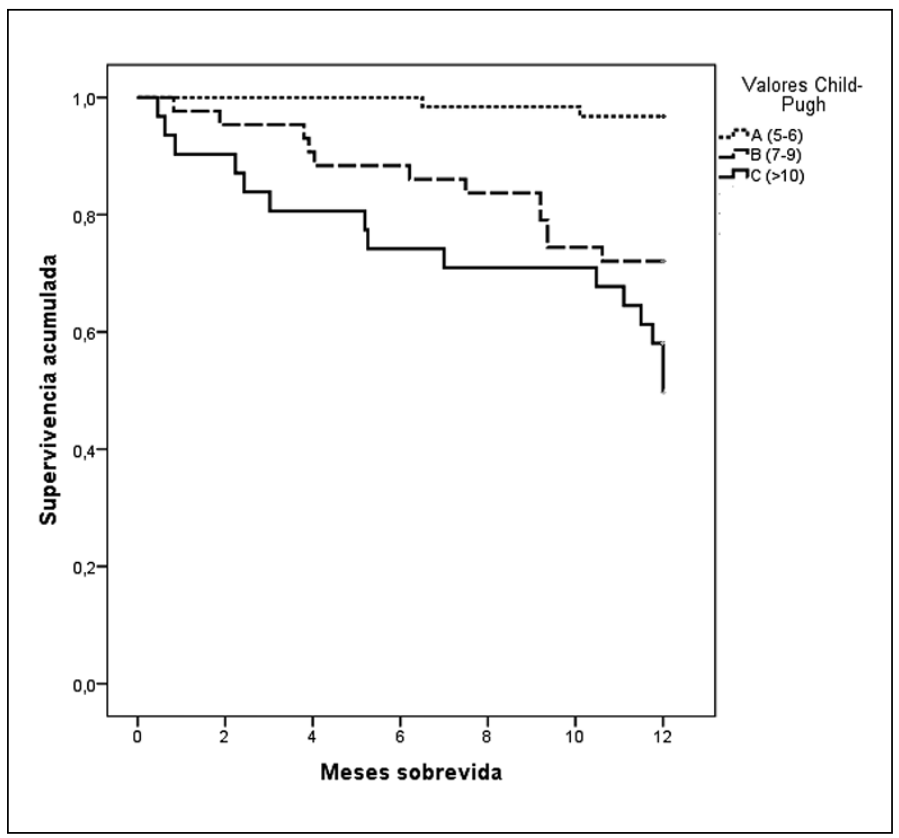

Figura 2. Sobrevida del grupo según $C P$.

ascitis). Por otro lado, el uso de la creatinina en la clasificación de MELD implica asumir cierta variabilidad, ya que su nivel es dependiente de la masa muscular y estado nutricional ${ }^{32}$. En consecuencia, al usar MELD podríamos estar subvalorando el pronóstico de estos pacientes.

En este trabajo se compararon las clasificacio- nes de CP y MELD para predecir la sobrevida al año, en pacientes chilenos. Es un estudio multicéntrico, con un universo mixto, público y privado, de distintas áreas de la región metropolitana, que se llevó a cabo en 3 instituciones de salud (dos del sistema público y una privada), por lo cual podría ser considerado una muestra representativa de la 


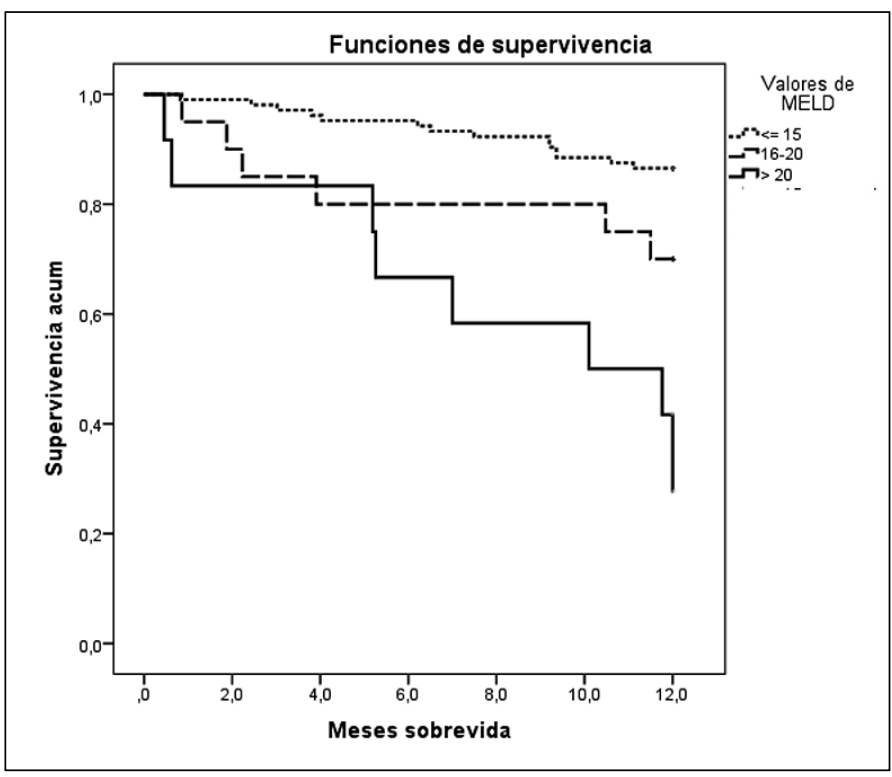

Figura 3. Sobrevida del grupo según MELD.

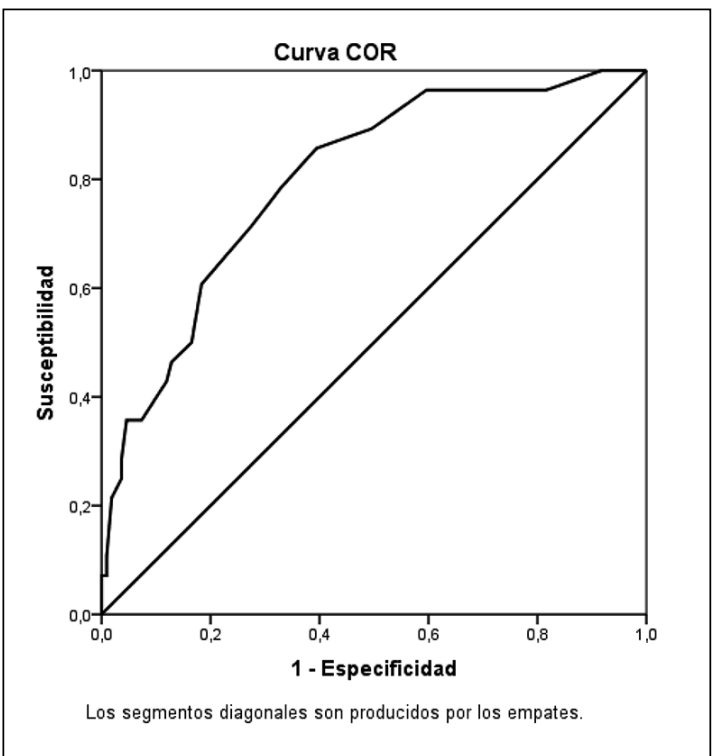

Figura 4. Curva de análisis ROC: grupo MELD.

población. Las tres instituciones contaban además con la posibilidad de manejar adecuadamente la cirrosis y sus complicaciones, incluyendo manejo oportuno para hemorragia digestiva, encefalopatía hepática e infecciones, vigilancia por imágenes y posibilidad de derivación y enlistamiento para $\mathrm{TH}$ en caso necesario.

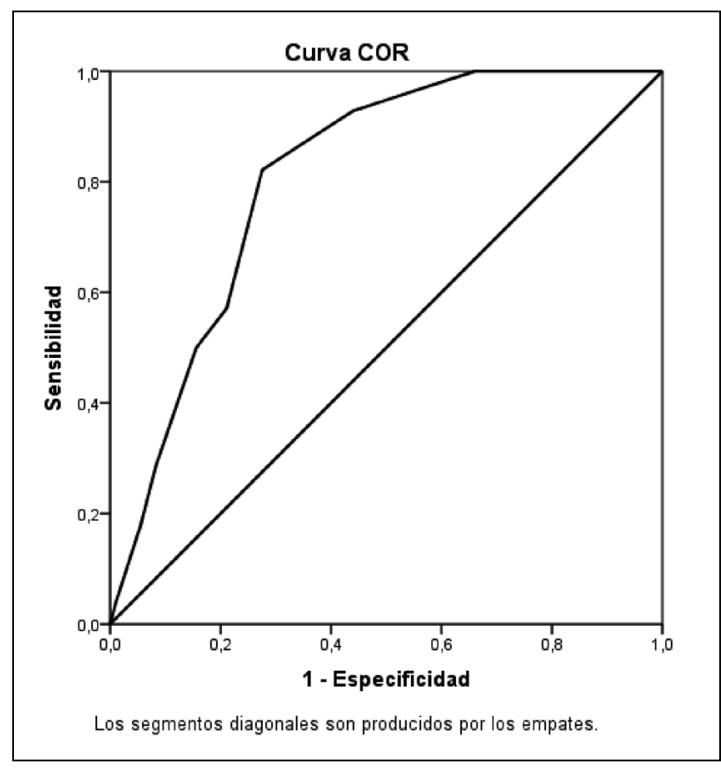

Figura 5. Curva de análisis ROC: grupoCHILD.

Los estudios internacionales han sido controversiales, mientras algunos de ellos muestran que MELD es mejor que CP en predecir sobrevi$\mathrm{da}^{27,33,23,6}$ otros consideran a la clasificación de CP superior o similar a MELD.

En nuestro trabajo, al igual que en otros estudios, la etiología más frecuente fue por alcohol, 
seguida en frecuencia por hepatitis $\mathrm{C}$ y esteatohepatitis no alcohólica. La mortalidad global de la cohorte fue de $20 \%$ al año de seguimiento. En ambas escalas se confirma que la mortalidad es mayor mientras más alta es la puntuación. Al comparar la sobrevida mediante las curvas de Kaplan Meier en la escala de MELD, se observa que no existen diferencias significativas entre los grupos de menor y mayor severidad. El área bajo la curva (AUROC) es 0,80 para MELD y 0,81 para la escala CP. Este resultado nos permite inferir que, el uso del sistema MELD para establecer el pronóstico no implica una gran diferencia en el alistamiento para TH, y permite validarlo como un sistema fiable al contar con variables más objetivas, para la inclusión en lista de espera de trasplante y asignación de órganos en nuestro país.El sistema MELD utiliza exámenes de laboratorio, simples y objetivos (bilirrubina, INR y creatinina) que están disponibles en todas las instituciones del país. De esta forma, se mantiene como una escala más objetiva, dinámica, de fácil uso y más reproducible al disminuir la variabilidad interobservador de la escala $\mathrm{CP}^{34}$, entre sus limitaciones está el no considerar complicaciones de la cirrosis como hemorragia digestiva o encefalopatía ${ }^{35,36}$.

Entre las limitaciones de este estudio, consideramos que podría influir el tamaño de la muestra y las posibles diferencias en la técnica utilizada para medir la creatinina.

En conclusión, nuestros resultados sugieren que ambas clasificaciones son comparables en relación a su capacidad de predecir sobrevida, lo cual valida el uso del sistema MELD como sistema pronóstico de cirrosis en Chile, pudiendo ser una herramienta confiable en la asignación de órganos y TH en nuestro país.

\section{Referencias}

1. Mokdad AA, López AD, Shahraz S, Lozano R, Mokdad $\mathrm{AH}$, Stanaway J, et al. Liver cirrhosis mortality in 187 countries between 1980 and 2010: a systematic analysis. BMC Med [Internet] 2014; 12 (1): 145. Available from: http://www.biomedcentral.com/1741-7015/12/145.

2. Keeffe EB. Liver Transplantation: Current Status and Novel Approaches to Liver Replacement. Gastroenterology [Internet] 2001 [citado el 11 de junio de 2013]; 120 (3): 749-62. Available from: http://linkinghub.elsevier. com/retrieve/pii/S0016508501630026.
3. Innocenti F, Hepp J, Zapata R, Sanhueza E, Humeres R, Ríos $\mathrm{H}$, et al. A decade of adult liver transplantation in a single center in Chile. Transplant Proc [Internet] 2004; 36 (6): 1673-4. Available from: http://www.ncbi.nlm. nih.gov/pubmed/15350448.

4. Murray KF, Carithers RL. AASLD Practice Guidelines: Evaluation of the Patient for Liver Transplantation. Hepatology [Internet]. 2005; 41 (6): 1-26. Available from: http://www.ncbi.nlm.nih.gov/pubmed/15880505.

5. Hepp J, Zapata R, Buckel E, Martínez J, Uribe M, Díaz JC, et al. [General considerations, indications and contraindications for liver transplantation in Chile: a multicenter consensus development document]. Rev Med Chile [Internet] 2008; 136 (6): 793-804. Available from: http://www.ncbi.nlm.nih.gov/pubmed/18769839.

6. Forman LM, Lucey MR. Predicting the prognosis of chronic liver disease: an evolution from child to MELD. Mayo End-stage Liver Disease. Hepatology [Internet]. 2001 [citado el 11 de agosto de 2014]; 33 (2): 473-5. Available from: http://www.ncbi.nlm.nih.gov/pubmed/11172352.

7. Wiesner RH. Patient selection in an era of donor liver shortage: current US policy. Nat Clin Pract Gastroenterol Hepatol [Internet] 2005 [citado el 28 de octubre de 2014]; 2 (1): 24-30. Available from: http://www.ncbi. nlm.nih.gov/pubmed/16265097.

8. Pugh RN, Murray-Lyon IM, Dawson JL, Pietroni MC, Williams R. Transection of the oesophagus for bleeding oesophageal varices. Br J Surg [Internet] 1973; 60 (8): 646-9. Available from: http://www.ncbi.nlm.nih.gov/ pubmed/4541913.

9. Reuben A. Child comes of age. Hepatology [Internet]. 2002 [citado el 4 de noviembre de 2015]; 35 (1): 244-5. Available from: http://www.ncbi.nlm.nih.gov/pubmed/11786987.

10. Zhang F, Zhuge Y, Zou X, Zhang M, Peng C, Li Z, et al. Different scoring systems in predicting survival in Chinese patients with liver cirrhosis undergoing transjugular intrahepatic portosystemic shunt. Eur J Gastroenterol Hepatol [Internet]. 2014 [citado el 22 de noviembre de 2015]; 26 (8): 853-60. Available from: http://www.ncbi.nlm.nih.gov/pubmed/24915489.

11. Durand F, Valla D. Assessment of the prognosis of cirrhosis: Child-Pugh versus MELD. J Hepatol [Internet]. 2005 [citado el 25 de junio de 2013]; 42 Suppl (1): S100-7. Available from: http://www.ncbi.nlm.nih.gov/ pubmed/15777564.

12. Malinchoc M, Kamath PS, Gordon FD, Peine CJ, Rank J, ter Borg PC, et al. A Model to Predict Poor Survival in Patients Undergoing Transjugular Intrahepatic Portosystemic Shunts. Hepatology [Internet]. 2000; 31 (4): 
864-71. Available from: http://www.ncbi.nlm.nih.gov/ pubmed/10733541.

13. Wiesner R, Edwards E, Freeman R, Harper A, Kim $\mathrm{R}$, Kamath $\mathrm{P}$, et al. Model for end-stage liver disease (MELD) and allocation of donor livers. Gastroenterology [Internet]. 2003; 124 (1): 91-6. Available from: http:// www.ncbi.nlm.nih.gov/pubmed/12512033.

14. Wiesner RH, McDiarmid SV, Kamath PS, Edwards EB, Malinchoc M, Kremers WK, et al. MELD and PELD: application of survival models to liver allocation. Liver Transpl [Internet]. 2001; 7 (7): 567-80. Available from: http://www.ncbi.nlm.nih.gov/pubmed/11460223.

15. Kamath PS, Wiesner RH, Malinchoc M, Kremers W, Therneau TM, Kosberg CL, et al. A model to predict survival in patients with end-stage liver disease. Hepatology [Internet]. 2001; 33 (2): 464-70. Available from: http://www.ncbi.nlm.nih.gov/pubmed/11172350.

16. Freeman RB, Wiesner RH, Edwards E, Harper A, Merion $\mathrm{R}$, Wolfe $\mathrm{R}$, et al. Results of the first year of the new liver allocation plan. Liver Transpl [Internet]. 2004 Jan [citado el 28 de agosto de 2016]; 10 (1): 7-15. Available from: http://www.ncbi.nlm.nih.gov/pubmed/14755772.

17. Said A, Williams J, Holden J, Remington P, et al. Model for end stage liver disease score predicts mortality across a broad spectrum of liver disease. J Hepatol [Internet]. 2004 [citado el 21 de septiembre de 2015]; 40 (6): 897 903. Available from: http://www.ncbi.nlm.nih.gov/ pubmed/15158328.

18. Botta F, Giannini E, Romagnoli P, Fasoli A, Malfatti F, Chiarbonello B, et al. MELD scoring system is useful for predicting prognosis in patients with liver cirrhosis and is correlated with residual liver function: a European study. Gut [Internet]. 2003; 52 (1): 134-9. Available from: http://www.ncbi.nlm.nih.gov/pubmed/12477775.

19. Dunn W, Jamil LH, Brown LS, Wiesner RH, Kim WR, Menon KVN, et al. MELD accurately predicts mortality in patients with alcoholic hepatitis. Hepatology [Internet]. 2005; 41 (2): 353-8. Available from: http://www. ncbi.nlm.nih.gov/pubmed/15660383.

20. Brown RS, Lake JR. The survival impact of liver transplantation in the MELD era, and the future for organ allocation and distribution. Am J Transplant [Internet]. 2005 [citado el 21 de septiembre de 2015]; 5 (2): 203-4. Available from: http://www.ncbi.nlm.nih.gov/pubmed/15643978.

21. Kremers WK, van IJperen M, Kim WR, Freeman RB, Harper AM, Kamath PS, et al. MELD score as a predictor of pretransplant and posttransplant survival in OPTN/UNOS status 1 patients. Hepatology [Internet]. 2004; 39 (3): 764-9. Available from: http://www.ncbi. nlm.nih.gov/pubmed/14999695.
22. Christensen E. Prognostic models including the Child-Pugh, MELD and Mayo risk scores-where are we and where should we go? J Hepatol [Internet]. 2004 Aug [citado el 22 de noviembre de 2015]; 41 (2): 344-50. Available from: http://www.ncbi.nlm.nih.gov/ pubmed/15288486.

23. Attia K, Ackoundou-N'guessan K, N'dri-yoman A, Mahassadi A, Messou E, Bathaix Y, et al. Child-Pugh-Turcott versus Meld score for predicting survival in a retrospective cohort of black African cirrhotic patients. World J Gastroenterol 2008; 14 (2): 286-91. Available from: http://www.ncbi.nlm.nih.gov/pubmed/18186569.

24. Cholongitas E, Papatheodoridis GV, Vangeli M, Terreni N, Patch D, Burroughs AK. Systematic review: The model for end-stage liver disease-should it replace Child-Pugh's classification for assessing prognosis in cirrhosis? Aliment Pharmacol Ther [Internet]. 2005; 22 (11-12): 1079-89. Available from: http://www.ncbi.nlm. nih.gov/pubmed/16305721.

25. Boursier J, Cesbron E, Tropet A-L, Pilette C. Comparison and improvement of MELD and Child-Pugh score accuracies for the prediction of 6-month mortality in cirrhotic patients. J Clin Gastroenterol [Internet]. 2009; 43 (6): 580-5. Available from: http://www.ncbi.nlm.nih. gov/pubmed/19197195.

26. Huo T-I, Wu J-C, Lin H-C, Lee F-Y, Hou M-C, Lee P-C, et al. Evaluation of the increase in model for end-stage liver disease (DeltaMELD) score over time as a prognostic predictor in patients with advanced cirrhosis: risk factor analysis and comparison with initial MELD and Child-Turcotte-Pugh score. J Hepatol [Internet]. 2005; 42 (6): 826-32. Available from: http://www.ncbi.nlm. nih.gov/pubmed/15885353.

27. Huo, TI, Lin HC, Wu JC, Lee FY, Hou MC, Lee PC, et al. Proposal of a modified Child-Turcotte-Pugh scoring system and comparison with the model for end-stage liver disease for outcome prediction in patients with cirrhosis. Liver Transpl 2006; 12: 65-71. doi:10.1002/ lt. 20560 .

28. Hoteit M-A, Ghazale A-H, Bain A-J, Rosenberg E-S, Easley K-A, Anania F-A, et al. Model for end-stage liver disease score versus Child score in predicting the outcome of surgical procedures in patients with cirrhosis. World J Gastroenterol [Internet]. 2008 [citado el 21 de septiembre de 2015]; 14 (11): 1774-80. Available from: http://www.pubmedcentral.nih.gov/articlerender.fcgi?artid=2695918\&tool=pmcentrez\&rendertype $=$ abstract.

29. Peng Y, Qi X, Guo X. Child-Pugh Versus MELD Score for the Assessment of Prognosis in Liver Cirrhosis: A Systematic Review and Meta-Analysis of Observational Studies. Medicine (Baltimore) [Internet]. 2016 [citado el 
1 de septiembre de 2016]; 95 (8): e2877. Available from: http://www.ncbi.nlm.nih.gov/pubmed/26937922.

30. Díaz LA, Robles C, Norero B, Elgueta S, Humeres R, Poniachik J, et al. Sistema MELD para la priorización de trasplante hepático en Chile: resultados a 3 años desde su inicio. Gastroenterol Latinoam 2015; 26 (4): 86-8.

31. Hepp J, Innocenti FA. Liver transplantation in Latin America: Current status. Transplantation Proceedings, Volume 36, Issue 6, July-August 2004, Pages 1667-8, ISSN 0041-1345, http://dx.doi.org/10.1016/j.transproceed.2004.06.021.

32. Gunsar F, Raimondo ML, Jones S, Terreni N, Wong C, Patch D, et al. Nutritional status and prognosis in cirrhotic patients. Alimentary Pharmacology \& Therapeutics 2006; 24: 563-72. doi:10.1111/j.1365-2036.2006. 03003.x.

33. Papatheodoridis GV, Cholongitas E, Dimitriadou E, Touloumi G, Sevastianos V, Archimandritis AJ. MELD vs Child-Pugh and creatinine-modified Child-Pugh score for predicting survival in patients with decompensated cirrhosis. World J Gastroenterol WJG [Internet]. 2005; 11 (20): 3099-104. Available from: http://www. ncbi.nlm.nih.gov/pubmed/15918197.

34. D’Amico G, García-tsao G, Pagliaro L, Amico GD. Natural history and prognostic indicators of survival in cirrhosis: A systematic review of 118 studies. J Hepatol [Internet] 2006; 44 (1): 217-31. Available from: http:// www.ncbi.nlm.nih.gov/pubmed/16298014.

35. Durand F, Valla D. Assessment of prognosis of cirrhosis. Semin Liver Dis [Internet]. 2008 [citado el 1 de septiembre de 2016]; 28 (1): 110-22. Available from: http:// www.ncbi.nlm.nih.gov/pubmed/18293281.

36. Huo T-I, Lin H-C, Wu J-C, Hou M-C, Lee F-Y, Lee P-C, et al. Limitation of the model for end-stage liver disease for outcome prediction in patients with cirrhosis-related complications. Clin Transplant [Internet]. 2006 [citado el 11 de agosto de 2014]; 20 (2): 188-94. Available from: http://www.ncbi.nlm.nih.gov/pubmed/16640525. 